\title{
Analysis of KRAP expression and localization, and genes regulated by KRAP in a human colon cancer cell line
}

\author{
Takahiro Fujimoto $\cdot$ Midori Koyanagi · \\ Iwai Baba · Kazuhiko Nakabayashi · Norihiro Kato • \\ Takehiko Sasazuki · Senji Shirasawa
}

Received: 29 May 2007 / Accepted: 18 September 2007/Published online: 13 October 2007

(C) The Japan Society of Human Genetics and Springer 2007

\begin{abstract}
We previously identified the human KRAP (Ki-ras-induced actin-interacting protein) gene from the cDNA library of human colon cancer HCT116 cells as one of the genes whose expression levels were up-regulated by activated Ki-ras. Although the KRAP gene is structurally conserved from fish to mammalian species, the expression pattern and function of KRAP still remain to be elucidated. Here, we have generated a specific polyclonal antibody for KRAP and characterized the histological expression of KRAP in mouse tissues. KRAP was ubiquitously expressed in mouse tissues, with high levels in pancreas, liver, and brown adipose tissues, and KRAP was co-localized with filamentous actin along the apical membranes in both pancreas and liver tissues. A subfractionation study
\end{abstract}

Electronic supplementary material The online version of this article (doi:10.1007/s10038-007-0204-8) contains supplementary material, which is available to authorized users.

T. Fujimoto $\cdot$ M. Koyanagi - I. Baba $\cdot$ K. Nakabayashi ·

S. Shirasawa $(\square)$

Department of Cell Biology, Faculty of Medicine,

Fukuoka University, 7-45-1 Nanakuma, Jonan-ku,

Fukuoka 814-0180, Japan

e-mail: sshirasa@fukuoka-u.ac.jp

K. Nakabayashi

Department of Maternal-Fetal Biology,

National Center for Child Health and Development,

2-10-1 Okura, Setagaya-ku, Tokyo 157-8535, Japan

N. Kato

Department of Gene Diagnostics and Therapeutics,

Research Institute, International Medical Center of Japan,

1-21-1 Toyama, Shinjuku-ku, Tokyo 162-8655, Japan

T. Sasazuki

International Medical Center of Japan, 1-21-1 Toyama,

Shinjuku-ku, Tokyo 162-8655, Japan revealed that KRAP is a cytoplasmic protein and that the majority is associated with the cytoskeleton. Furthermore, microarray gene expression profile by inhibiting KRAP expression in HCT116 cells showed that several receptors and signal molecules frequently deregulated in cancers were differentially expressed in the KRAP-knockdown cells. All of these results suggested that KRAP might be a cytoskeleton-associated protein involving the structural integrity and/or signal transductions in human cancers.

Keywords KRAP · F-actin - Cytoskeletal protein . Pancreas · Liver - Acinar cells · Bile canaliculi . Microarray

\section{Introduction}

The human KRAP (Ki-ras-induced actin-interacting protein) gene was originally identified as one of the genes whose expression levels were up-regulated by activated Ki-ras in human colon cancer HCT116 cells (Inokuchi et al. 2004). While KRAP was rarely expressed in normal colon epithelium, deregulated constitutive KRAP expression was observed in some cancer cells (Inokuchi et al. 2004). In humans, KRAP mRNA was most strongly expressed in the pancreas and testis, with ubiquitous distribution among the other organs at the lower levels (Inokuchi et al. 2004). KRAP is structurally predicted to possess a coiled-coil motif within its $\mathrm{C}$ terminus and exogenous KRAP expression was localized along the actin stress fibers in NIH3T3 cells (Inokuchi et al. 2004), suggesting that the KRAP might be involved in the regulation of F-actin. KRAP is structurally well conserved from fish to mammalian species (UCSC Genome Browser) and mouse KRAP has high amino acid sequence identity with 
human KRAP, together suggesting that KRAP may play physiologically critical roles. Here, as the first step toward understanding the functional role of KRAP, we generated a polyclonal antibody specific for this protein and investigated the tissue distribution and cellular expression of KRAP by northern blotting, in situ hybridization, western blotting, and immunohistochemical staining in mouse tissues and cells. Furthermore, we analyzed the microarray gene expression profile by inhibiting KRAP expression in HCT116 cells.

\section{Materials and methods}

Animals

All of the animals used in this study were treated in accordance with the rules of Fukuoka University, Japan.

Northern blot analysis

Total RNA was extracted from C57BL/6J mouse adult tissues with Trizol (Invitrogen, Carlsbad, CA, USA) according to the manufacturer's protocol. The total RNA $(15 \mu \mathrm{g})$ was electrophoresed in $0.9 \%$ agarose/formaldehyde gel and transferred onto a nylon membrane. After fixation by UV crosslinking, the membrane was hybridized with mouse KRAP cDNA (GenBank accession no. AB120565), as described previously (Okumura et al. 1999).

Generation of polyclonal antiserum

Recombinant human KRAP (amino acids 1039-1246) was expressed as a bacterial fusion protein using the pGEX6P-1 vector (Amersham Pharmacia). The fusion protein was soluble in nondenaturing buffer and was purified with Glutathione Sepharose 4B (Amersham Pharmacia). Antiserum was obtained by injecting the recombinant KRAP protein into Japanese White Rabbit, followed by booster injection. Antiserum was purified with an affinity column prepared by cross-linking the recombinant protein to CNBr-activated Sepharose 4B (Amersham Pharmacia).

Cell culture

HCT 116 cells and $\mathrm{HKe} 3$ cells were cultured at $37^{\circ} \mathrm{C}$ with $5 \% \mathrm{CO}_{2}$ in DMEM containing $10 \%$ fetal calf serum (FCS), as described previously (Okumura et al. 1999). NIH3T3 cells were maintained at $37^{\circ} \mathrm{C}$ with $5 \% \mathrm{CO}_{2}$ in DMEM containing $10 \%$ FCS and penicillin-streptomycin- glutamine. NIH3T3 cells were transfected with a small inhibitory RNA (siRNA) using Lipofectamine 2000 (Invitrogen) according to the manufacturer's protocol. Two distinct siRNAs were designed to target the coding region of the mouse KRAP gene (nucleotides 729-753 or 19701994, GenBank accession no. AB120565). Scrambled RNAs containing the same number of each nucleotide as the siRNAs targeting the KRAP gene were used as controls. The following siRNA duplexes were used in this study: KRAP \#1, 5'-C CUG ACU ACU GUG GCC AAU GCA UUU-3' and $3^{\prime}-\mathrm{G}$ GAC UGA UGA CAC CGG UUA CGU AAA-5'; scramble RNA \#1, 5'-C CUC AUC GUG UAC CGC GUA AAG UUU- $3^{\prime}$ and $3^{\prime}-\mathrm{G}$ GAG UAG CAC AUG GCG CAU UUC AAA- $5^{\prime}$; KRAP \#2, $5^{\prime}$-C CAC ACA CCA UAU UCU CAG AUC CUU-3' and $3^{\prime}-\mathrm{G}$ GUG UGU GGU AUA AGA GUC UAG GAA-5'; scramble RNA \#2, 5'-C CAA CCC UAU ACU CUG AUA CAC CUU-3' and $3^{\prime}-\mathrm{G}$ GUU GGG AUA UGA GAC UAU GUG GAA-5'.

\section{RT-PCR}

Total RNA was extracted from NIH3T3 cells $48 \mathrm{~h}$ after the transfection of $K R A P$-specific siRNAs as described above. RT-PCR was done with Superscript III (Invitrogen) and LA $\mathrm{Taq}^{\mathrm{TM}}$ Polymerase (Takara), using primers for KRAP, $5^{\prime}-\mathrm{C}$ ATATGACAGAGGAGGACA-3' and $5^{\prime}$-GTGGCTGTCC TGCTTAGG-3'; and $\beta$-actin, $5^{\prime}$-ATGGATGACGATATC GCTGCG-3' and 5'-GAAGCTGTAGCCACGCTCGG-3'.

\section{Western blot analysis}

C57BL/6J mouse tissues or cultured cells were lysed in RIPA buffer [50 mM Tris-HCl, pH 7.5, $150 \mathrm{mM} \mathrm{NaCl,}$ $1 \%$ NP-40, $0.5 \%$ deoxycholate, $0.1 \%$ SDS, protease inhibitor cocktail (Roche)] and subjected to western blotting, as described previously (Okumura et al. 1999). Antibodies used for western blotting were as follows: affinity purified anti-KRAP antibodies (dilution 1:2,000), anti-ERK1 antibody (K-23; Santa Cruz Biotechnology; 1:1,000), anti-actin antibody (H-300; Santa Cruz Biotechnology; 1:1,000), anti-CREB antibody (Cell Signaling; 1:500), anti-E-cadherin antibody (BD Biosciences; $1: 1,000)$, and anti-Radixin antibody (C-15; Santa Cruz Biotechnology; 1:500).

In situ hybridization

An adult ICR mouse was perfused with $10 \%$ neutral formalin and the pancreas was dissected. Then, the pancreas was embedded in paraffin. Tissue sections $(4 \mu \mathrm{m})$ were 
dewaxed and hybridized as described previously (Hoshino et al. 1999). A 510-bp DNA fragment corresponding to the nucleotide positions $3250-3759$ of mouse KRAP cDNA (GenBank accession no. AB120565) in pBluescript II $\mathrm{SK}(+)$ was used for the generation of digoxigenin-labeled sense or antisense RNA probes. The expressions were detected using NBT (nitro-blue tetrazolium chloride)BCIP (5-bromo-4-chloro- $3^{\prime}$-indolylphosphatase $p$-toluidine salt) and tissue slides were counterstained with Kernechtrot stain solution (Hoshino et al. 1999).

\section{Immunohistochemistry}

C57BL/6J mouse tissues were frozen in an ethanol/dry ice bath, followed by the preparation of sections $(6 \mu \mathrm{m})$ using a cryomicrotome. Sections were fixed with $4 \%$ paraformaldehyde in $0.1 \mathrm{M}$ phosphate buffer for $30 \mathrm{~min}$ at room temperature (RT), blocked with $5 \%$ bovine serum, $0.1 \%$ Tx-100, $150 \mathrm{mM} \mathrm{NaCl}, 50 \mathrm{mM}$ Tris- $\mathrm{HCl}, \mathrm{pH} 7.5$ for $30 \mathrm{~min}$ at RT, and then subjected to double-immunostaining with rabbit polyclonal anti-KRAP antibodies (dilution 1:400) and mouse monoclonal anti-insulin antibody: clone K36aC10 (Sigma-Aldrich; 1:2,000). Primary antibodies were visualized with goat anti-rabbit $\operatorname{IgG}$ conjugated to FITC or goat anti-mouse IgG conjugated to Alexa Fluor 594. F-actin was visualized with rhodaminephalloidin (molecular probe) according to the manufacturer's protocol. Fluorescence images were acquired by confocal fluorescence microscopy, as described previously (Inokuchi et al. 2004).

\section{Subcellular fractionation}

The preparation of cytoplasmic and nuclear fractions was performed as described previously (Joo et al. 2004).

Subcellular fractionation of C57BL/6J mouse liver was performed using a compartmental protein extraction kit (Chemicon) according to the manufacturer's protocol. In this assay, cytoplasmic, membrane, and cytoskeletal fractions were sequentially obtained from liver homogenate. Proportional amounts of each fraction were analyzed by western blotting.

Gene expression profile by inhibiting KRAP expression in HCT116 cells

HCT116 cells were transfected with a small inhibitory RNA (siRNA) using MicroPorator MP-100 (Digital Bio) according to the manufacturer's protocol. Three distinct siRNAs were designed to target the coding region of the human KRAP gene (nucleotides 1179-1203, 1524-1548, or 1732-1756, GenBank accession no. AB116937). Scrambled RNAs containing the same number of each nucleotide as the siRNAs targeting the KRAP gene were used as controls. The following siRNA duplexes were used in this study: KRAP \#1, $5^{\prime}$-G GAG AAU GCU GAU AGU GAU AGA AUU- $3^{\prime}$ and $3^{\prime}-\mathrm{C}$ CUC UUA CGA CUA UCA CUA UCU UAA- $5^{\prime}$; scramble RNA \#1, $5^{\prime}$-G GAC GUA UAG UGU GAG AUA AAG AUU- $3^{\prime}$ and $3^{\prime}-\mathrm{C}$ CUG CAU AUC ACA CUC UAU UUC UAA-5'; KRAP \#2, 5'-C CAG CUA GGU CUU ACG AAG UCG AAA- $3^{\prime}$ and $3^{\prime}$-G GUC GAU CCA GAA UGC UUC AGC UUU- ${ }^{\prime}$; scramble RNA \#2, $5^{\prime}$-C CAU AGG UCU UAC GAA GUC GGC AAA- $3^{\prime}$ and $3^{\prime}-\mathrm{G}$ GUA UCC AGA AUG CUU CAG CCG UUU-5'; KRAP \#3, 5'-C CAA CAG CAC AAG ACC AGC CUU AUU- $3^{\prime}$ and $3^{\prime}$-G GUU GUC GUG UUC UGG UCG GAA UAA-5'; scramble RNA \#3, 5'-C CAG AAC ACG ACA ACC GUC UCA AUU- $3^{\prime}$ and $3^{\prime}-\mathrm{G}$ GUC UUG UGC UGU UGG CAG AGU UAA-5'. Twenty-four hours after the transfection, the total RNA was isolated from $1 \times 10^{6}$ cells with the RNeasy Mini Kit (Qiagen). Biotinylated antisense cRNA was prepared using GeneChip Expression 3'-Amplification One-Cycle cDNA Synthesis kit and GeneChip Expression 3'-Amplification Reagent (Affymetrix) according to the manufacturer's protocol. Biotinylated cRNAs were fragmented and hybridized to Affymetrix GeneChip Human Genome U133Plus2.0 arrays. The arrays were washed and scanned on a GeneChip Fluidics Station 450 and GeneChip Scanner Model 3000 controlled by a GeneChip Operating System v1.4 (GCOS) (Affymetrix) according to standard Affymetrix protocols. The data were annotated according the NetAffx database (http://www. affymetrix.com/analysis/index.affx) as of July 2007. Three pairs of comparisons of the gene expression profile between KRAP-knockdown cells and the control cells (KRAP \#1 vs. scramble \#1, KRAP \#2 vs. scramble \#2, and KRAP \#3 vs. scramble \#3) was performed. A cut-off value of 1.5-fold or more change between the KRAP-knockdown $(\mathrm{KD})$ and the control $(\mathrm{Ctl})$ cells was used.

\section{Results and discussion}

Tissue distribution of the mouse KRAP mRNA and protein

To reveal the tissue distribution of the mouse KRAP transcript, a northern blot analysis was performed on the adult mouse tissues (Fig. 1a). KRAP mRNA were strongly expressed in the forebrain, liver, kidney, white adipose tissue (WAT), and brown adipose tissue (BAT), whereas it was weakly expressed in the heart and skeletal muscle tissues. Next, to examine the exact tissue distribution of the 


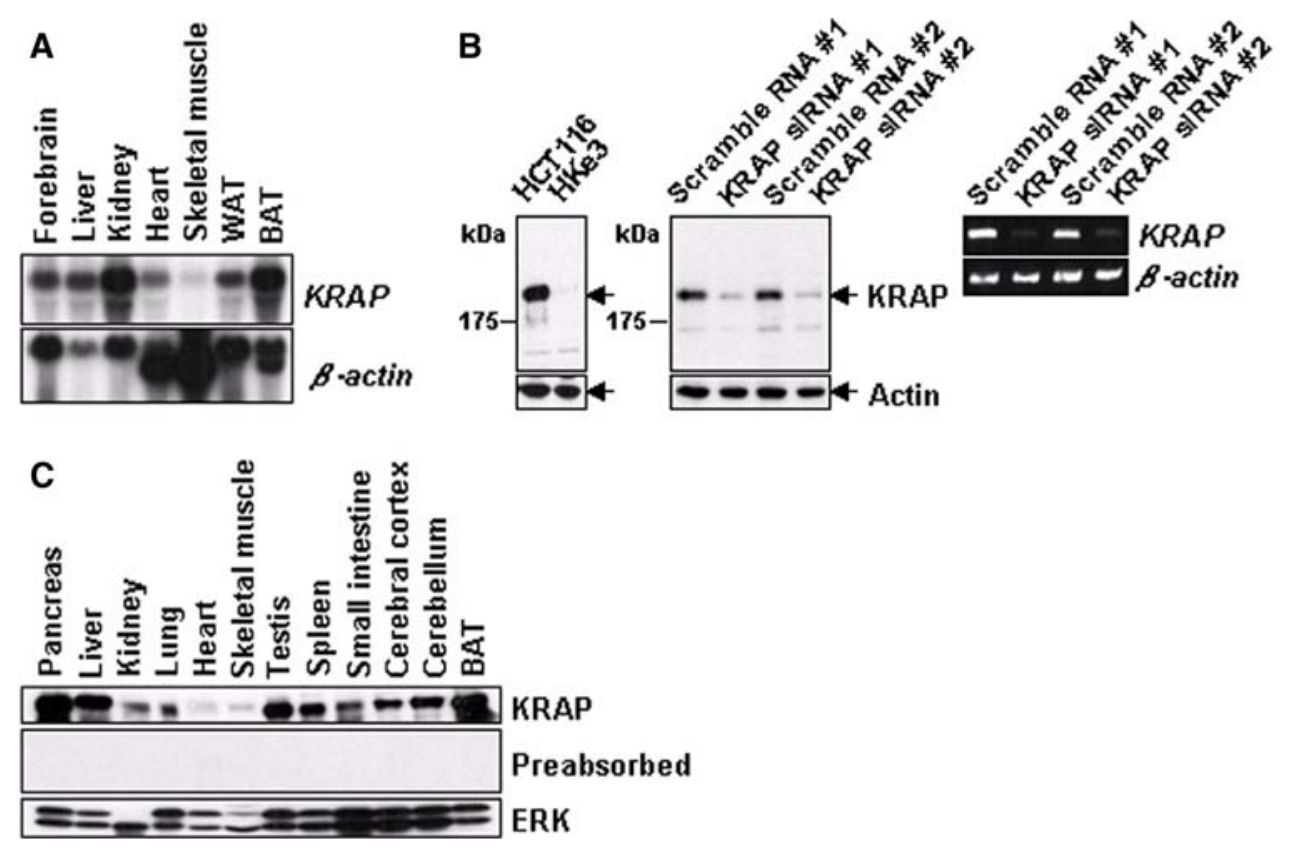

Fig. 1a-c Tissue distribution of the mouse KRAP mRNA and protein. a Northern blot analysis of the KRAP transcript. Total RNA was extracted from seven kinds of adult mouse tissues and northern blotting was carried out using the probes of KRAP (upper panel) and $\beta$-actin (lower panel). b Detection of KRAP protein by western blotting using the anti-KRAP polyclonal antibody. KRAP expression in HCT116 and HKe3 cells (left panel). KRAP protein (middle panel) and mRNA (right panel) expressions in NIH3T3 cells

$48 \mathrm{~h}$ after the transfection of KRAP-specific siRNAs. Scramble RNAs were used as the transfection control. c Western blot analysis for KRAP expression in the 12 kinds of adult mouse tissues. The specificity of the band was confirmed using anti-KRAP preabsorbed with immunizing recombinant protein (middle panel). Western blotting using anti-ERK antibody was done as a loading control (lower panel)

KRAP protein, a polyclonal antibody was generated by immunizing a recombinant KRAP protein in a rabbit. To validate the reactivity and specificity of the KRAP polyclonal antibody, HCT116 cells and HKe3 cells disrupted at activated Ki-ras by homologous recombination (Shirasawa et al. 1993) were examined using this KRAP antibody. A $180-\mathrm{kDa}$ band was evidently detected in HCT116 cell extracts (Fig. 1b), whereas the corresponding band in HKe3 cell extracts was rarely detected (Fig. 1b). Furthermore, in NIH3T3 mouse cells, KRAP was evidently detected as a 180 -kDa protein (Fig. 1b), while the immunoreactive band was dramatically reduced in NIT3T3 cells treated with siRNAs for KRAP (Fig. 1b). The RT-PCR analysis of KRAP mRNA expression in NIH3T3 cells treated with the siRNAs for KRAP validated the specificities of the siRNAs used in this study (Fig. 1b). These results together indicated that the antibody generated here specifically recognizes both human and mouse KRAP proteins. Then, western blot analysis using this anti-KRAP polyclonal antibody was performed against the homogenates from various tissues of adult mice. Strong expressions of KRAP were detected in the pancreas, liver, brown adipose, and testis, and moderate expressions were detected in the kidney, lung, spleen, small intestine, and

brain (Fig. 1c). On the other hand, KRAP was rarely detected in the heart and skeletal muscle tissues (Fig. 1c). The relative amount of KRAP protein among the mouse tissues was well correlated with the results from the northern blot analysis (Fig. 1a). Taken together, KRAP is an ubiquitous protein in adult mouse tissues.

Regional and cellular localization of KRAP

In situ hybridization was performed to reveal the distribution of the KRAP transcript in the adult mouse pancreas. $K R A P$ was restricted to the exocrine acinar region of the pancreas and no significant signals were observed in the islet region (Fig. 2a, c). To examine the precise regional and cellular localization of the KRAP protein, we carried out immunohistochemical analysis on the mouse pancreas and liver sections. In the pancreas, KRAP staining was observed in the exocrine acinar cells, while the signals were not detected in the insulin-positive islet region (Fig. 3a-c), confirming the previous result obtained by in situ hybridization that the KRAP transcript was restricted to the exocrine acinar region in the pancreas (Fig. 2a, c). Double immunohistochemical staining using the KRAP 
Fig. 2a-d In situ hybridization analysis of KRAP mRNA expression. A section of an adult mouse pancreas was hybridized with the KRAP antisense RNA probe (a and $\mathbf{c})$. Control hybridization was performed with the sense probe on consecutive sections (b and $\mathbf{d}$ ). The scale bar represents $100 \mu \mathrm{m}$

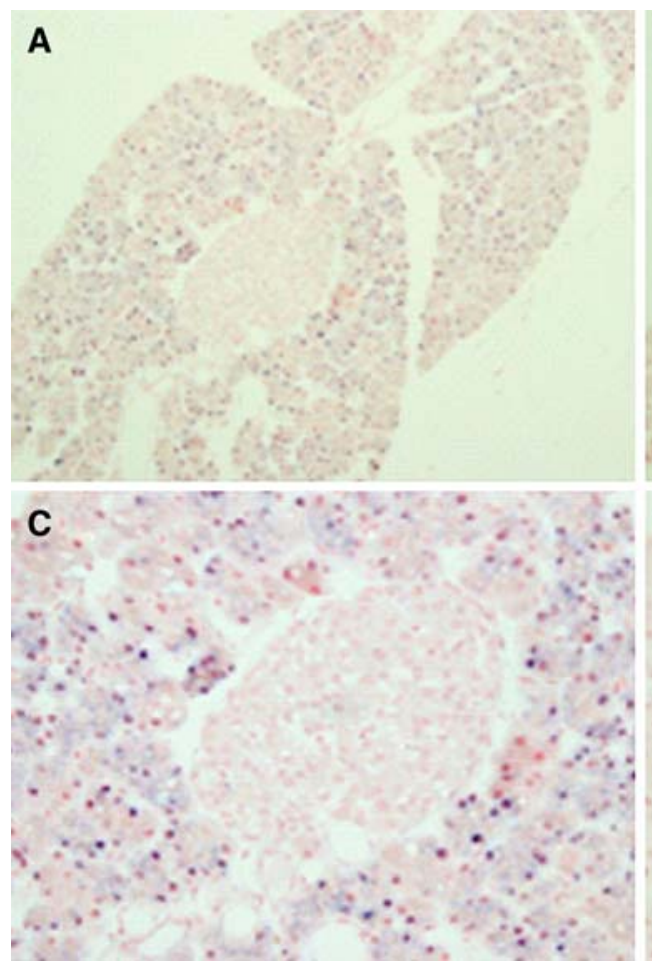

B

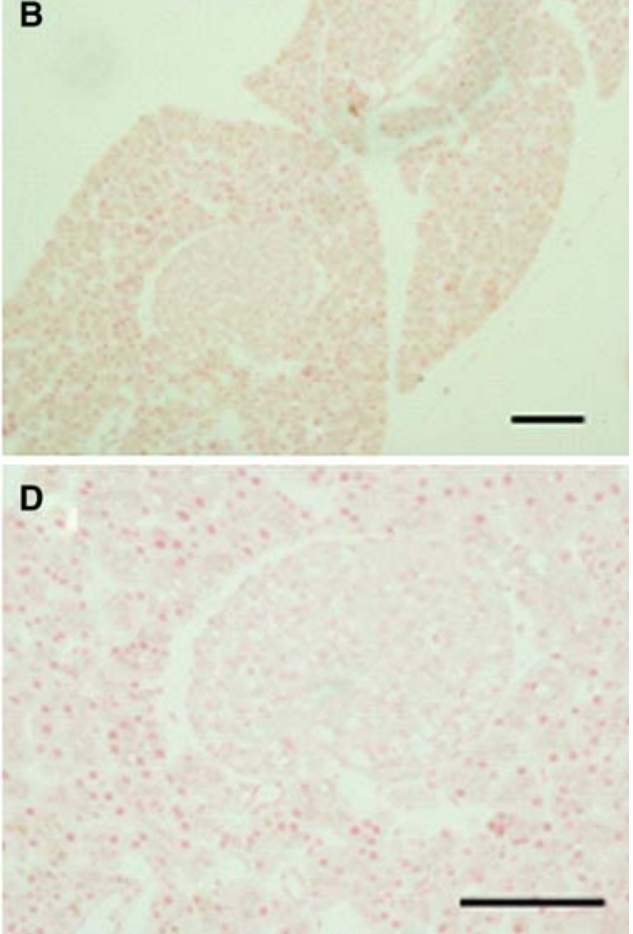

antibody and phalloidin showed that KRAP was co-localized with F-actin along the apical membrane (Fig. 3d-f). In the liver, KRAP was detected as a belt-like localization along the bile canalicular membrane of hepatocytes and was co-localized with F-actin (Fig. 3g-i). These cellular localizations were correlated with the former observation that ectopically expressed KRAP protein was localized along the F-actin in NIH3T3 cells (Inokuchi et al. 2004). Furthermore, the common feature of the KRAP localization along the apical membrane in the two distinct tissues suggested the possibility that KRAP might be localized in a similar fashion to other tissues.

\section{Subcellular distribution of KRAP}

The subcellular fractionation of NIH3T3 cells was carried out to understand the localization of KRAP. A cytoplasmic protein-ERK (p44/42 MAP kinase) and a nuclear proteinCREB (cyclic-AMP response element-binding) were expectedly fractionated into the cytoplasmic and nuclear fractions, respectively (Fig. 4a). In this condition, KRAP was exclusively detected in the cytoplasmic fraction. Next, the subcellular localization of KRAP was examined by the biochemical fractionation of mouse liver tissue. ERK and an integral protein-E-cadherin were expectedly fractionated into the cytoplasmic and membrane fractions, respectively (Fig. 4b). In this condition, KRAP was detected in the cytoplasmic fraction and the majority of KRAP protein was concentrated in the cytoskeletal fraction (Fig. 4b), suggesting that KRAP is a cytoplasmic protein associated with the cytoskeleton. Radixin is an adaptor protein concentrated in the apical membranes in hepatocytes (Kikuchi et al. 2002; Fouassier et al. 2001). In our assay, Radixin was expectedly detected in the cytoskeletal fraction, but the significant amount of this protein was readily solubilized in the cytoplasmic fraction compared with KRAP (Fig. 4). All of these results suggested the possibility that KRAP may be a constituent of cytoskeleton-associated proteins anchoring integral and/or signal proteins.

Gene expression profile by inhibiting KRAP expression in HCT116 cells

To reveal the functions of KRAP, KRAP expression was inhibited using siRNAs and microarray gene expression analysis was performed in a colon cancer cell line, HCT116. The treatment of HCT116 cells with three distinct siRNAs specific for KRAP sequence led to a significant decrease in KRAP protein expression $(81.4 \pm 2.2 \%)$ compared to scramble RNA-treated controls (Fig. 5a). For the microarray gene expression analysis, a cut-off value of 1.5 -fold or more change between the KRAP-knockdown (KD) and the control (Ctl) cells was used. A total of 113 probesets on the microarray were commonly changed in the three pairs of comparisons. Out of 113 probesets, 70 and 43 were up- and down-regulated 
Fig. 3a-i

Immunohistochemical localization of KRAP in the mouse pancreas and liver. Immunofluorescence photomicrographs for KRAP (a and d), insulin (b), F-actin (e), and merged images $(\mathbf{c}$ and $\mathbf{f})$ in the pancreas.

Immunofluorescence photomicrographs for KRAP (g), F-actin (h), and merged images (i) in the liver. The scale bar represents $50 \mu \mathrm{m}$
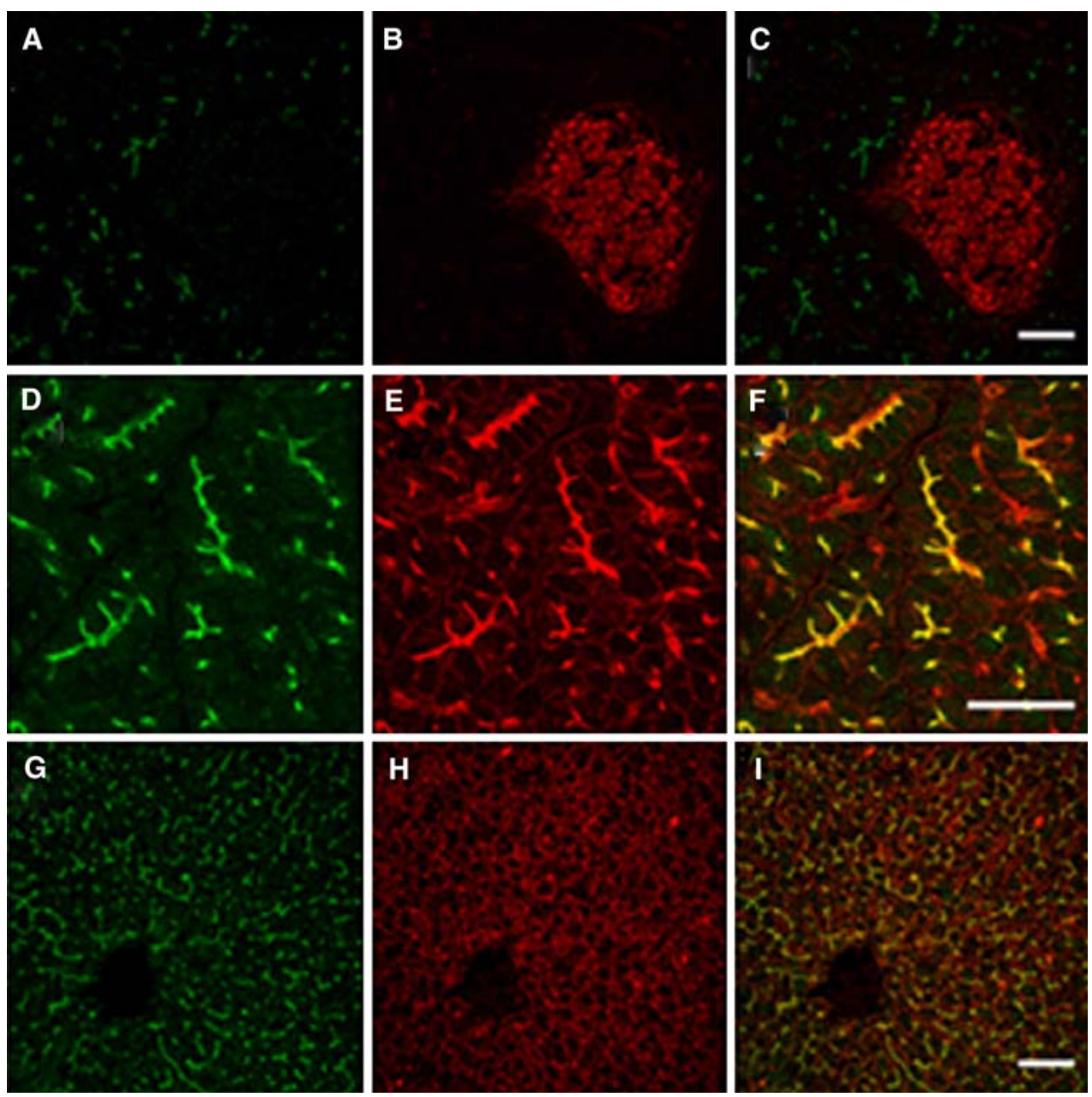

H

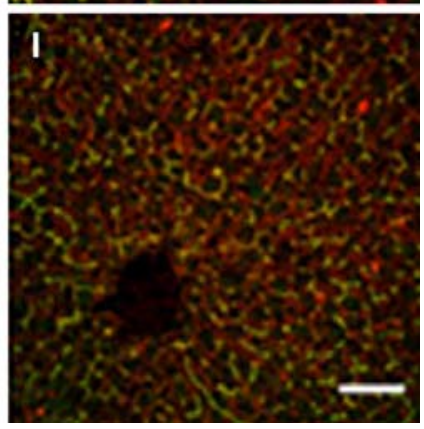

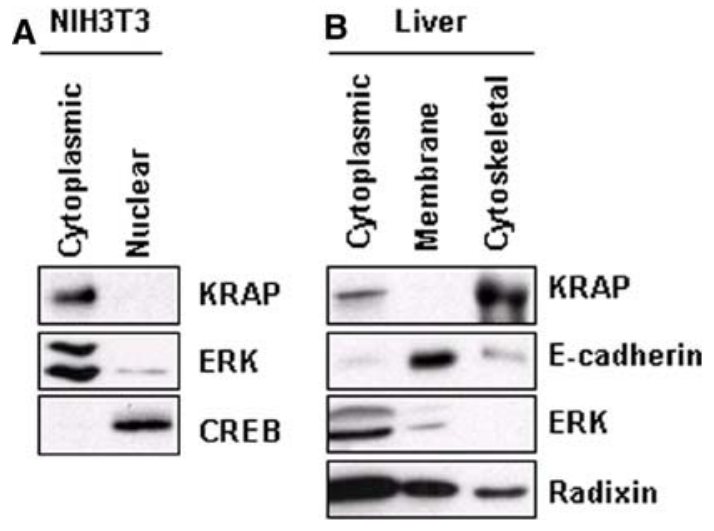

Fig. 4a, b Subcellular distribution of KRAP. a NIH3T3 cells were fractionated into cytoplasmic and nuclear fractions. b Mouse liver homogenate was fractionated into cytoplasmic, membrane, and cytoskeletal fractions. Proportional amounts of each fraction were analyzed by western blotting using anti-KRAP, anti-CREB, anti-E-cadherin, anti-ERK, and anti-Radixin antibodies

in KD cells, respectively (Fig. 5b). The expression data for these probesets are provided in the supplementary material (Supplementary Table S1). Two distinct probesets for KRAP (236207_at, 202506_at) were down-regulated by
siRNA treatments, showing the reduction of KRAP expression (Supplementary Table S1).

Gene ontology analysis by GeneSpring GX 7.3.1 showed that genes with differential expression levels between $\mathrm{KD}$ and $\mathrm{Ctl}$ cells were associated with protein kinase $\mathrm{C}$ activation, cellular morphogenesis, lipoprotein catabolism, inositol phosphate-mediated signaling, G-protein signaling, the regulation of MAPK activity, and cellsurface-receptor-linked signal transduction (Supplementary Table S2), suggesting that KRAP expression might affect the cellular malignancy by altering the diverse signaling pathways. Among the differentially expressed genes (Supplementary Table S1), $\alpha_{V}$ integrin, $\beta_{1}$ ntegrin, GNAS complex locus (GNAS), mitogen-activated protein kinase kinase kinase (MAP4K3), interleukin 6 receptor (IL6R), interleukin 6 signal transducer (IL6ST), interleukin $1 \beta$ convertase (CASP1), and EPH receptor B2 (EPHB2) were remarkably interesting from the viewpoint of the cancer biology. Among the integrin receptors, $\alpha_{V} \beta_{1}, \alpha_{3} \beta_{1}, \alpha_{5} \beta_{1}$, and $\alpha_{6} \beta_{4}$ integrins are associated with enhanced cell motility and cancer metastasis (Felding-Habermann 2003). One of the important functions of the $\beta_{1}$ integrin is to 
A

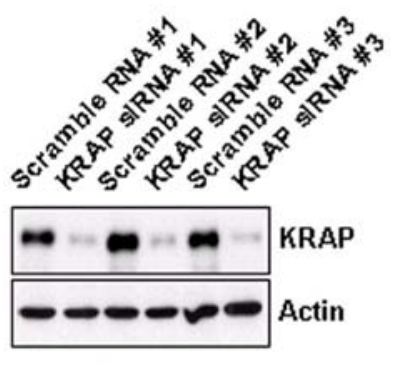

$B$ Up-regulated in KD cells

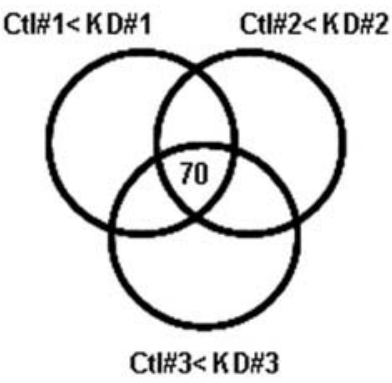

Down-regulated in KD cells

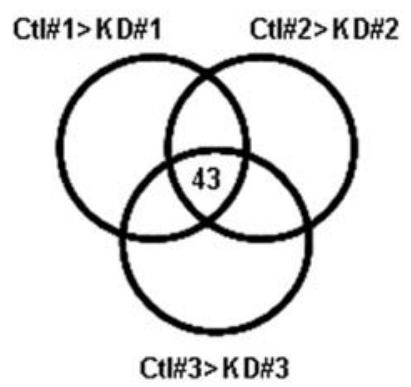

Fig. 5a, b Gene expression profile by inhibiting KRAP expression in HCT 116 cells. a KRAP protein expression in HCT116 cells $24 \mathrm{~h}$ after the transfection of three distinct $K R A P$-specific siRNAs. Scramble RNAs were used as the transfection controls. b Venn diagram showing the up-regulated 70 probesets and down-regulated 43 probesets in KRAP-knockdown $(K D)$ cells. A cut-off value of

suppress apoptosis in mammary epithelial cells through the negative regulation of interleukin $1 \beta$ convertase (CASP1) (Boudreau et al. 1995). Both $\beta_{1}$ integrin and CASPl were changed in KD cells in our analysis (Supplementary Table S1), suggesting that there might be a functional interaction between $\beta_{1}$ integrin and CASP1 in HCT116 cells. Loss- and gain-of-function mutations of GNAS involved in the cyclic AMP-dependent pathway cause human diseases (Weinstein et al. 2006). MAP4K3 is a component in the nutrient-responsive pathway affecting cell growth (Findlay et al. 2007). Deregulated expression of various EPH receptors, including EPHB2, has been reported in diverse tumor types (Guo et al. 2006; Jubb et al. 2005). All of these results suggested that KRAP might play critical roles in cancer-associated signaling pathways, including Ras, MAPKs (MAP kinases), integrins, EPH receptors, and protein kinase $\mathrm{C}$.

In this study, we demonstrated that KRAP was ubiquitously expressed in mouse tissues and was associated with cytoskeletal fraction, and the microarray-based gene expression analysis revealed that KRAP affected particular receptors and signal molecules involved in cellular malignancy. These findings together suggested that KRAP may influence the cellular events related to attachment, migration, proliferation, and apoptosis. Further analyses of KRAP should be awaited for the exact understanding of KRAP functions in cancer cells.

Acknowledgments This study was supported in part by the Program for Promotion of Fundamental Studies in Health Sciences of the National Institute of Biomedical Innovation (NIBI).

\section{References}

Boudreau N, Sympson CJ, Werb Z, Bissell MJ (1995) Suppression of ICE and apoptosis in mammary epithelial cells by extracellular matrix. Science 267:891-893 1.5-fold or more change between the $\mathrm{KD}$ and the control $(\mathrm{Ctl})$ cells was used. The up-regulated genes included $\alpha_{V}$ integrin, $\beta_{1}$ ntegrin, GNAS, MAP4K3, IL6R, and IL6ST. The down-regulated genes included $C A S P 1$ and $E P H B 2$. The data of the differentially expressed genes is provided in Supplementary Table S1

Felding-Habermann B (2003) Integrin adhesion receptors in tumor metastasis. Clin Exp Metastasis 20:203-213

Findlay GM, Yan L, Procter J, Mieulet V, Lamb RF (2007) A MAP4 kinase related to Ste20 is a nutrient-sensitive regulator of mTOR signalling. Biochem J 403:13-20

Fouassier L, Duan CY, Feranchak AP, Yun CH, Sutherland E, Simon F, Fitz JG, Doctor RB (2001) Ezrin-radixin-moesin-binding phosphoprotein 50 is expressed at the apical membrane of rat liver epithelia. Hepatology 33:166-176

Guo DL, Zhang J, Yuen ST, Tsui WY, Chan ASY, Ho C, Ji J, Leung SY, Chen X (2006) Reduced expression of EphB2 that parallels invasion and metastasis in colorectal tumors. Carcinogenesis 27:454-464

Hoshino M, Sone M, Fukata M, Kuroda S, Kaibuchi K, Nabeshima Y, Hama C (1999) Identification of the stef gene that encodes a novel guanine nucleotide exchange factor specific for Rac1. J Biol Chem 274:17837-17844

Inokuchi J, Komiya M, Baba I, Naito S, Sasazuki T, Shirasawa S (2004) Deregulated expression of KRAP, a novel gene encoding actin-interacting protein, in human colon cancer cells. J Hum Genet 49:46-52

Joo A, Aburatani H, Morii E, Iba H, Yoshimura A (2004) STAT3 and MITF cooperatively induce cellular transformation through upregulation of c-fos expression. Oncogene 23:726-734

Jubb AM, Zhong F, Bheddah S, Grabsch HI, Frantz GD, Mueller W, Kavi V, Quirke P, Polakis P, Koeppen H (2005) EphB2 is a prognostic factor in colorectal cancer. Clin Cancer Res 11:51815187

Kikuchi S, Hata M, Fukumoto K, Yamane Y, Matsui T, Tamura A, Yonemura S, Yamagishi H, Keppler H, Tsukita S, Tsukita S (2002) Radixin deficiency causes conjugated hyperbilirubinemia with loss of Mrp2 from bile canalicular membranes. Nat Genet 31:320-325

Okumura K, Shirasawa S, Nishioka M, Sasazuki T (1999) Activated Ki-Ras suppresses 12-O-tetradecanoylphorbol-13-acetateinduced activation of the c-Jun NH2-terminal kinase pathway in human colon cancer cells. Cancer Res 59:2445-2450

Shirasawa S, Furuse M, Yokoyama N, Sasazuki T (1993) Altered growth of human colon cancer cell lines disrupted at activated Ki-ras. Science 260:85-88

Weinstein LS, Chen M, Xie T, Liu J (2006) Genetic diseases associated with heterotrimeric $\mathrm{G}$ proteins. Trends Pharmacol Sci 27:260-266 